\title{
Enriched environment treatment reverses depression-like behavior and restores reduced hippocampal neurogenesis and protein levels of brain-derived neurotrophic factor in mice lacking its expression through promoter IV
}

\author{
S Jha, B Dong and K Sakata
}

Promoter IV-driven expression of brain-derived neurotrophic factor (BDNF), a major neuronal growth factor, is implicated in the pathophysiology of major depression. We previously reported that mice lacking expression of BDNF through promoter IV (BDNF-KIV mice) exhibit a depression-like phenotype. Here, we examined whether the depression-like phenotype and decreased levels of BDNF because of promoter IV deficit could be rescued by enriched environment (EE) treatment, a potential antidepressant intervention. Three weeks of EE treatment rescued depression-like behavior of BDNF-KIV mice as assessed by the tail suspension test, open-field test and sucrose preference test. EE treatment also increased BDNF transcripts driven by multiple endogenous promoters and restored BDNF protein levels in the hippocampus (HIP) of BDNF-KIV mice. Further, we investigated adult hippocampal neurogenesis as a possible cellular mechanism underlying the depression-like behavior and its recovery in BDNF-KIV mice. We found that the number of surviving progenitors and their dendritic length in the dentate gyrus of the HIP were reduced in BDNF-KIV mice compared with the control wild-type mice. EE treatment restored the reduction in cell survival and dendritic length and increased cell proliferation in BDNF-KIV mice. In conclusion, this study demonstrated that EE rescued depression-like behavior, decreased BDNF levels and defective neurogenesis in the HIP caused by lack of promoter IV-driven BDNF expression. These results suggest that decreased BDNF levels because of one impaired promoter can be compensated by other BDNF promoters and that BDNF levels may be one of the key factors regulating depression and antidepressant effects through hippocampal neurogenesis.

Translational Psychiatry (2011) 1, e40; doi:10.1038/tp.2011.33; published online 13 September 2011

\section{Introduction}

Brain-derived neurotrophic factor (BDNF) is a major neuronal growth factor in the brain that promotes neurogenesis, neuronal maturation and synaptic plasticity. ${ }^{1,2}$ BDNF has been implicated in both the pathophysiology of major depression and the actions of antidepressants. ${ }^{3}$ However, the lack of clear-cut depression-like behavior with manipulation of BDNF levels (knockout (KO) and knockdown) in an animal model has delayed studies connecting BDNF and depression in vivo. ${ }^{3}$ Recently, we showed direct evidence that supports the BDNF hypothesis of depression by focusing on an endogenous BDNF promoter; mice that lack promoter IV-driven BDNF expression ${ }^{4}$ and have reduced levels of BDNF in the brain displayed depression-like behavior. ${ }^{5}$ Although the Bdnf gene is regulated by at least nine different promoters, ${ }^{6}$ promoter IV (previously classified as promoter III) is most responsive to neuronal activity and induces activity-dependent expression of BDNF. ${ }^{7-9}$ This finding suggests the intriguing hypothesis that increased neuronal activity induces activity-dependent BDNF expression, which further induces, through a feedback mechanism, neuronal activity to maintain active brain function. Any disruption to activity-dependent BDNF expression would lead to decreased neuronal activity and function, which could lead to depression. Decreased function of BDNF promoter IV may occur in real life via reduced neuronal stimuli, mutations in the promoter region and epigenetic processes through stress. Supporting this, studies have shown that social dominant stress and immobilization stress decrease the function of promoter IV through epigenetic regulation processes. ${ }^{10,11}$

However, questions remain. Can the depression-like behavior caused by impaired promoter IV-driven BDNF be rescued by antidepressant interventions? If so, what are the underlying mechanisms? We addressed these questions by using BDNF knock-in IV (BDNF-KIV) mice, which have a unique feature: functional expression of BDNF protein via one

Department of Pharmacology, University of Tennessee Health Science Center, Memphis, TN, USA

Correspondence: Dr K Sakata, Department of Pharmacology/Psychiatry, College of Medicine, University of Tennessee Health Science Center, 874 Union Avenue, Room 430 Memphis, TN 38163, USA.

E-mail: ksakata@uthsc.edu

Keywords: BDNF; promoter; neurogenesis; enriched environment; depression; mice

Received 21 June 2011; accepted 8 July 2011 
specific promoter (promoter IV) is ablated by insertion of a green fluorescent protein gene, but other promoters and the coding region of BDNF are kept intact (for details, see Sakata et al. ${ }^{4}$ ). We hypothesized that one promoter deficiency could be compensated by other intact promoters in the $B d n f$ gene. Different types of antidepressant interventions, which include antidepressant medications, electroconvulsive therapy, exercise and enriched environment (EE), have been reported to increase expression of BDNF transcripts, notably in the hippocampus (HIP) through different promoters. ${ }^{12-16}$ Although BDNF induction by these interventions (approximately a 1.2- to 3-fold increase over several weeks) is not as robust as the one caused by neural depolarization or seizure through promoter IV (usually more than a 4-fold increase within $3 \mathrm{~h}$ after administering stimuli), ${ }^{17}$ accumulation of a small increase in BDNF protein may provide an antidepressant effect. The antidepressant effect of BDNF has been long suggested by evidence that direct infusion of BDNF into the HIP produces antidepressant effects in behavioral models of depression. ${ }^{18}$ If decreased BDNF expression leads to depression, an increase in BDNF levels should reverse the depression-like behavior and pathophysiology of BDNF-KIV mice. However, this hypothesis-reduced BDNF levels lead to depression and depression-like behavior while restoration of BDNF levels has antidepressant effects-has not yet been tested, most likely because no animal model with reduced BDNF levels clearly shows depression-like behavior ${ }^{3,19}$ until recently. ${ }^{5,20}$ Here, we addressed whether treatment with an antidepressant intervention could reverse the depression-like behavior and reduced BDNF levels in BDNF-KIV mice. An EE ( $>3$ weeks) that includes a running wheel has been reported to increase BDNF levels through multiple promoters (I, II, III, IV and VI) ${ }^{16}$ and to produce antidepressant effects. ${ }^{21-23}$ Therefore, we used EE as a potential intervention to upregulate BDNF levels by endogenous multiple promoters.

Further, we investigated a possible cellular mechanism underlying depression-like behavior and its recovery in BDNF-KIV mice. One of the hypothesized cellular mechanisms underlying major depression and its recovery is hippocampal neurogenesis. ${ }^{24}$ Neurogenesis in the dentate gyrus (DG) of the HIP is decreased by stress ${ }^{25}$ and increased by antidepressant interventions including EE. ${ }^{26-28}$ This downand upregulation of neurogenesis are paralleled by changes in BDNF expression levels; BDNF levels, in particular, promoter IV-driven BDNF transcripts, are downregulated by different types of stress, ${ }^{10,11,29-34}$ while different BDNF promoter-driven BDNF levels are upregulated by many antidepressant interventions including $\mathrm{EE}$ in the HIP. ${ }^{12-15}$ Exogenous BDNF has been shown to increase neurogenesis in the DG. ${ }^{2}$ Thus, it has been suggested that BDNF may regulate neurogenesis and, through this process, might mediate the effects of stress and AD interventions. ${ }^{35}$ Here, we examined whether promoter IV-driven BDNF expression may be required for regulating basal levels of neurogenesis in the HIP by using BDNF-KIV mice and investigated whether the observed phenotype in adult neurogenesis could be reversed by EE treatment. The levels of proliferation, survival and differentiation of progenitors were examined.

\section{Subjects and methods}

Animals. The generation of BDNF-KIV mice has been described previously. ${ }^{4}$ Male adult age-matched (2-5 monthold) BDNF-KIV (KIV) and control wild-type (WT) littermates were group-housed (four to five animals per cage) for 3 weeks in either standard conditions (SCs) (regular cage; $27 \times 16 \times 12 \mathrm{~cm})$ or EE conditions. EE consisted of a larger cage $(44 \times 22 \times 16 \mathrm{~cm})$ containing a plastic running wheel, nesting material, and an assortment of toys with different colors and textures (igloo, dome, balls, tunnels, etc., Bio-Serve, Frenchtown, NJ, USA). Once a week, the toys were changed, and mice in EE were given rodent Foraging Crumble (Bio-Serve). All animals were housed in a normal 12:12 h dark-light cycle and had ad libitum access to food and water. All animal experiments were approved by the University of Tennessee Laboratory Animal Care and Use Committee.

Behavioral tests. The open-field test (OFT), tail suspension test (TST), and sucrose preference test (SPT), were conducted as described previously ${ }^{5}$ with modifications. In the previous SPT, two bottles were placed on the one side of a cage grid, making one bottle closer to the center and, therefore more accessible. In this study, both bottles were placed toward the center of the cage and were equally distributed to prevent difference in bottle accessibility. Immobility time in the TST was measured by an observer blind to the experimental groups.

RNA extraction and quantitative reverse transcriptasePCR (qRT-PCR). Total RNA from each HIP was isolated using the RNeasy Kit (Qiagen, Valencia, CA, USA). In all, $1 \mu \mathrm{g}$ of total RNA was reverse-transcribed into single-stranded complementary DNA using the Transcriptor First Strand cDNA Synthesis Kit (Roche, Indianapolis, IN, USA) following the manufacturer's instructions. qRT-PCR was performed in a total volume of $5 \mu \mathrm{l}$ containing complementary DNA made from $5 \mathrm{ng}$ of total RNA, $1 \times$ TaqMan PCR master mix (Roche), $20 \mathrm{~nm}$ of each Universal Probe Library probe (Roche) and $40 \mathrm{~nm}$ of each primer. The sequences of primers and probes are shown in Supplementary Table 1. Amplification was carried out for 50 cycles using a Light Cycler 480 (Roche). Each cycle consisted of the following steps: $95^{\circ} \mathrm{C}$ for $10 \mathrm{~s}$, $60{ }^{\circ} \mathrm{C}$ for $30 \mathrm{~s}$ and $72^{\circ} \mathrm{C}$ for $10 \mathrm{~s}$. Relative gene expression values were determined using the $2^{-\Delta \Delta C T}$ method of Livak and Schmittgen ${ }^{36}$ with CyclophilinD as a reference gene.

BDNF enzyme-linked immunosorbent assay. Protein samples were prepared from the right HIP from each mouse, as described previously. ${ }^{4}$ BDNF protein levels were determined by enzyme-linked immunosorbent assay (BDNF Emax immunoassay system; Promega, Madison, WI, USA), and total protein concentrations were measured by DC protein assay (Bio-Rad Laboratories, Hercules, CA, USA), following the manufacturer's instructions. The BDNF signal was normalized to the total soluble protein in each sample.

Bromo deoxyuridine (BrdU) labeling and immunohistochemistry. BrdU labeling and immunohistochemistry were performed as described previously. ${ }^{37}$ Briefly, mice received a 
single intraperitoneal injection of BrdU (Fisher Scientific, Pittsburgh, PA, USA; $100 \mathrm{mg} \mathrm{kg}^{-1}$ of body weight) during the first 10 days or at the last day of the housing period in EE or SC. Immunohistochemistry detecting BrdU-positive (BrdU +) cells was performed $3 \mathrm{~h}$ or 2 weeks after the last injection of BrdU (Figure 3, top panels). Free-floating coronal sections ( $40 \mu \mathrm{m}$ for proliferation experiments and $50 \mu \mathrm{m}$ for survival experiments) were used for immunohistochemistry with rat anti-BrdU antibody (1:250; AbD Serotec, Raleigh, NC, USA) and goat anti-rat fluorescein isothiocyanate antibody (1:500; Millipore, Billerica, MA, USA). For double labeling, sections from the survival experiment were incubated with anti-BrdU antibody, then incubated with either mouse anti-NeuN (neuronal nuclei) antibody (1:500; Millipore) or rabbit antiGFAP (glial fibrillary acidic protein) antibody (1:1000; Millipore), followed by incubation with a cocktail of goat anti-rat fluorescein isothiocyanate antibody (1:500; Millipore) with either goat anti-mouse Alexa 555 antibody (1:500; Invitrogen, Carlsbad, CA, USA) or goat anti-rabbit Cy3 antibody (1:500; Invitrogen) for BrdU/NeuN or BrdU/GFAP, respectively. To detect doublecortin-positive $(\mathrm{DCX}+)$ cells, after blocking in $10 \%$ goat serum, sections were incubated with rabbit anti-DCX antibody (1:3000; Sigma, St Louis, MO, USA) for $4 \mathrm{~h}$ at room temperature, followed by incubation with goat anti-rabbit Cy3 antibody (1:500; Invitrogen).

TUNEL staining. For terminal deoxynucleotidyl transferasemediated dUTP nick-end labeling (TUNEL), sections were pretreated with $0.1 \mathrm{M}$ trisodium citrate $(\mathrm{pH} 6.0$, boiling in microwave) for $5 \mathrm{~min}$, and staining was performed using the Dead-End Fluorometric TUNEL System (Promega) following the manufacturer's instructions.

Quantitation. Images of the DG were acquired on an Olympus microscope (IX50, Center Valley, PA, USA) equipped with a video camera. BrdU + cells in the granule cell layer and the subgranule cell layer (SGZ) in the HIP were counted from these images. For double-labeling, at least $100 \mathrm{BrdU}+$ cells per animal were analyzed for the colocalization of both BrdU and the markers NeuN or GFAP on a confocal microscope (Nikon, Melville, NY, USA) with z-plane sectioning. To analyze DCX + cells, the number of $\mathrm{DCX}+$ cells and dendritic extension were measured in each DG. Dendritic extension was analyzed by counting the number of dendrites crossing at 50,100, 150 and $200 \mu \mathrm{m}$ from the SGZ. TUNEL-positive cells were counted using an Olympus microscope. Quantification of data was performed by an observer blinded to the genotype and experimental group.

Statistical analysis. Student's $t$-tests were performed on the two data groups. Two-way analysis of variance (ANOVA) was performed using Prism (GraphPad Software, La Jolla, CA, USA) for genotype effect and EE effect, and, when warranted, post hoc Bonferroni multiple comparisons were carried out. One-way ANOVA was performed with three or more data groups, followed by Bonferroni multiple comparisons. Data are presented as means \pm s.e. (standard error of mean). Statistical significance was set at ${ }^{\star} P<0.05$ and ${ }^{* *} P<0.01$.

\section{Results}

We examined the effects of the lack of promoter IV-driven BDNF expression by comparing BDNF-KIV and WT littermates in SC and EE conditions. We compared the effects of EE treatment on both genotypes by using two-way ANOVA. The statistical values are presented in Supplementary Table 2.

\section{EE reversed depression-like behavior in BDNF-KIV} mice. First, we examined whether the depression-like behavior of BDNF-KIV mice ${ }^{5}$ could be reversed by EE treatment at the end of the 3-week period in EE and SC by the OFT and TST. The OFT measures explorative locomotor activity in a novel open field, ${ }^{38}$ and the TST measures stressrelated despair (time of immobility) when mice are suspended by their tails. ${ }^{39}$ We replicated our previous report $^{4}$ that BDNF-KIV mice in SC displayed significantly reduced locomotor activity in the OFT $(P<0.01$, Figure 1a) without changing the time spent in the center $(P>0.05$, Supplementary Figure 1a) and significantly increased immobility in the TST $(P<0.05$, Figure $1 \mathrm{~b})$ compared with WT mice. When the effects of EE on the depression-like behavior was assessed by the OFT, two-way ANOVA revealed a significant effect of genotype $(P<0.05)$ and a trend toward a genotype-EE interaction $(P=0.053)$. The post hoc test showed that EE treatment significantly increased total activity of BDNF-KIV mice (KIV-EE vs KIV-SC, $P<0.05$; Figure 1a). There was no difference among the WT-SC, WT-EE and KIV-EE groups (Figure 1a, one-way ANOVA), which indicated that EE treatment reversed the reduced locomotor activity observed in BDNF-KIV mice, while it had no effect in WT mice. When effect of EE on total time spent in center was examined, twoway ANOVA revealed a significant effect of EE $(P<0.05)$. The post hoc test showed that EE treatment increased time in center in WT mice with a significance (WT-SC vs WT-EE, $P<0.05$ ) and in BDNF-KIV mice with a trend (KIV-SC vs KIV-EE, $P>0.05$ by Bonferroni multiple comparison; $P=0.03$ by Student's $t$-test, Supplementary Figure 1a). BDNF-KIV mice showed reduced frequency to enter the center compared with WT mice $(P<0.05)$, and this reduction was reversed be EE (Supplementary Figure 1b), mirroring the results of total activity in the OFT (Figure 1a). In the TST, EE significantly decreased the immobility time in both WT $(P<0.05)$ and BDNF-KIV $(P<0.01)$ mice (Figure $1 \mathrm{~b})$, indicating the antidepressant effect of EE in both genotypes. BDNF-KIV mice in EE exhibited no difference in the immobility time when compared with WT mice in SC $(P=0.6$; Figure $1 \mathrm{~b})$, indicating that the depression-like phenotype observed in BDNF-KIV mice in the TST was reduced by EE treatment. The results of OFT and TST suggested a possibility that EE treatment only increased locomotor activity rather than providing antidepressant effect. Therefore, we further conducted the SPT, which measures anhedonia (reduced preference to pleasurable sucrose water) and is locomotion-independent. ${ }^{40}$ We replicated our previous report ${ }^{4}$ that BDNF-KIV mice in SC displayed significantly reduced sucrose preference in the SPT $(P<0.01$, Figure 1c) compared with WT mice in SC. When 
effect of EE on SPT was examined, two-way ANOVA revealed a genotype-EE interaction $(P<0.01)$ and a trend toward a significant effect of genotype $(P=0.07)$. The post hoc test showed that EE treatment significantly increased percent sucrose consumption in BDNF-KIV mice (KIV-EE vs KIV-SC, $P<0.01$; Figure 1c). There was no difference among the WT-SC, WT-EE and KIV-EE groups by one-way
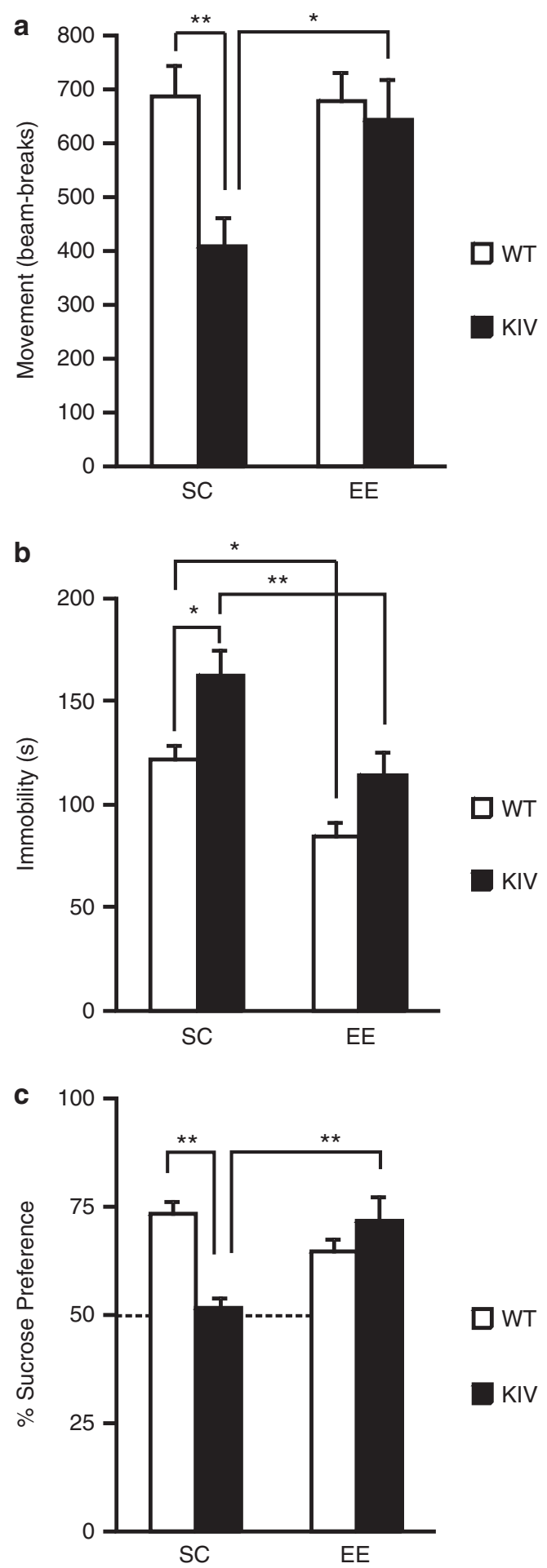

ANOVA (Supplementary Table 2), indicating that EE treatment rescued the reduced preference for sucrose observed in BDNF-KIV mice. The total liquid intake was significantly higher in WT-EE compared with other groups $(P<0.01)$ while no difference was observed among the other groups ( $P>0.05$, Supplementary Figure 2$)$.

EE increased expression of BDNF $m R N A$ and protein through multiple promoters. Although BDNF-KIV mice lack promoter IV-driven BDNF expression, they possess intact protein-coding sequence and other promoters for BDNF. ${ }^{4}$ We examined whether the antidepressant effects of EE were correlated to increased levels of BDNF mRNA through other BDNF promoters. When total BDNF mRNA levels were measured by expression levels of exon IX (the common BDNF protein-coding region) using qRT-PCR significant increases by $E E$ treatment were observed in both WT (43\% increase; $P<0.01$, Figure $2 \mathrm{a}$ ) and BDNF-KIV mice $(30 \%$ increase; $P<0.05$; Figure $2 a)$. When the contribution of each BDNF promoter was examined by measuring mRNA levels of respective exons, significant increases by EE were detected in exons I (101\% increase), Ila (61\% increase), Ilb (59\% increase), Ilc (62\% increase) and III (112\% increase) in WT-EE mice and in exon I $(66 \%$ increase) and exon III (88\% increase) in BDNF-KIV mice as compared with their counterparts in SC (Figure $2 b$, Supplementary Table 2). Taken together, these results indicate that EE treatment significantly induced BDNF mRNA expression through multiple promoters both in WT and BDNF-KIV mice.

Next, we measured the BDNF protein levels in the HIP by enzyme-linked immunosorbent assay to determine if increased BDNF mRNA levels by EE were translated into enhanced BDNF protein levels. Two-way ANOVA revealed significant effects of both genotype $(P<0.01)$ and $E E$ $(P<0.05)$ in BDNF protein levels. Post hoc tests revealed a significant decrease (35\%) in BDNF-KIV mice in SC compared with WT mice in SC $(P<0.01$; Figure 2c) and a significant increase (36\%) in BDNF-KIV mice by EE treatment (KIV-EE vs KIV-SC, $P<0.05$; Figure 2c). No significant difference was observed in WT-EE and KIV-EE mice compared with WT-SC $(P>0.05)$. These results indicate that

Figure 1 Effect of enriched environment (EE) on depression-like behavior in brain-derived neurotrophic factor knock-in IV (BDNF-KIV) and wild-type (WT) mice. (a) The open-field test measuring total locomotor activity of mice for $10 \mathrm{~min}$. Note that KIV-standard condition (SC) (BDNF-KIV mice in standard condition) showed reduced locomotor activity compared with WT-SC (WT mice in SC), while KIV-EE (BDNF-KIV mice in enriched environment) as well as WT-EE (WT mice in EE) showed similar locomotor activity compared to WT-SC ( $n=12$ per group). (b) The tail suspension test measuring immobility time of mice for $6 \mathrm{~min}$. Note the increased immobility time in KIV-SC but similar immobility time in KIV-EE compared with WTSC. ( $n=12$ per group). (c) The sucrose preference test measuring $\%$ sucrose preference (total intake of sucrose water/total liquid intake of sucrose water and water) of mice over $3 \mathrm{~h}$. The $50 \%$ dotted line is shown to indicate the equal preference for water and sucrose. Note that KIV-SC showed reduced sucrose preference compared with WT-SC, while KIV-EE as well as WT-EE showed sucrose preference similar to WT-SC ( $n=8$ per group). Results are expressed as mean \pm s.e. ${ }^{\star} P<0.05 ;{ }^{*} P<0.01$. 

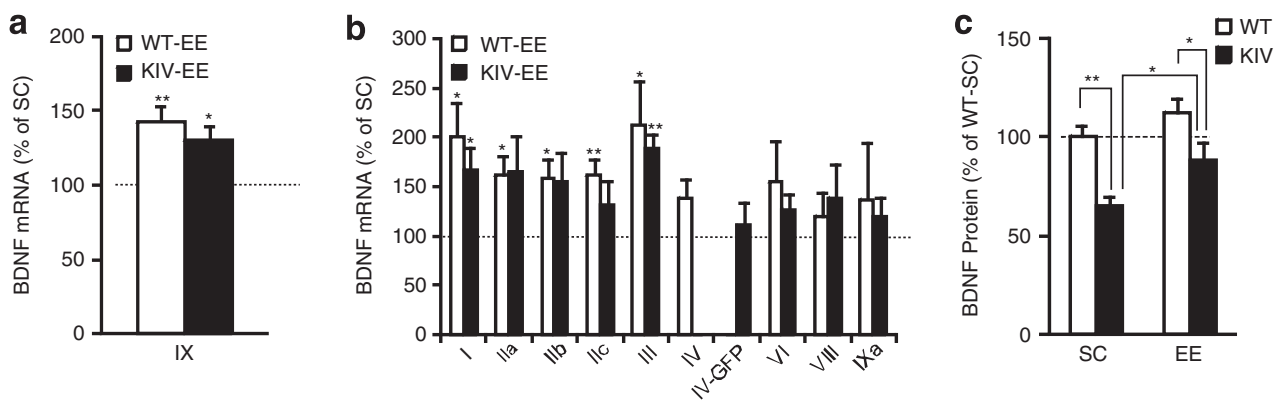

Figure 2 Effects of enriched environment (EE) on expression of brain-derived neurotrophic factor (BDNF) transcripts and protein levels in the hippocampus. (a) Quantitative reverse transcriptase (qRT)-PCR results showing total BDNF mRNA levels (protein-coding exon IX, $n=4$ per group). (b) qRT-PCR results showing mRNA levels of BDNF exons I, Ila, IIb, IIc, III, IV, VI, VIII and IXa in the hippocampus (HIP). Results are expressed as \% wild-type-standard condition (WT-SC) for WT-EE and \% knock-in IV (KIV)-SC for KIV-EE. Note that EE significantly increased levels of total BDNF mRNA levels in both genotypes through multiple promoters. ( $n=4$ per group). (c) Enzyme-linked immunosorbent assay (ELISA) quantification of BDNF protein levels in the HIP. Note that the BDNF protein levels were reduced in BDNF-KIV mice in SC compared with WT mice in SC. BDNF protein levels were increased in BDNF-KIV mice in EE compared with those in SC $\left(n=12\right.$ per group). ${ }^{*} P<0.05 ;{ }^{* \star} P<0.01$.

a
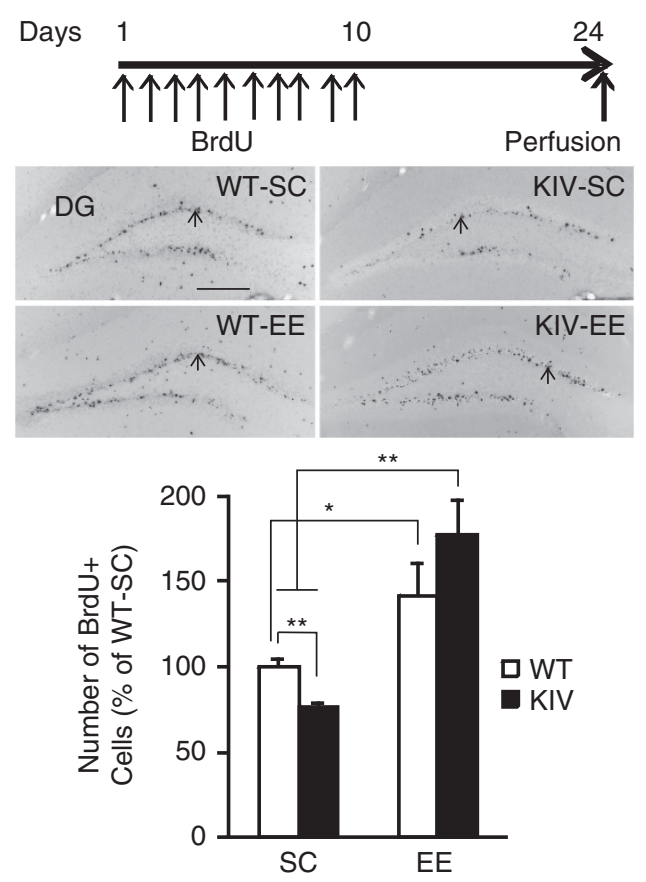

b

Days 1

SC or EE
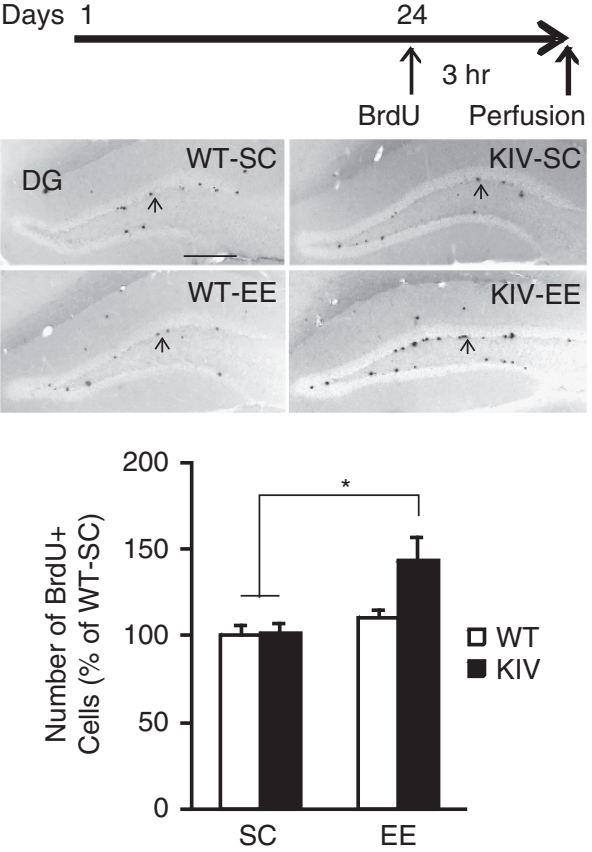

Figure 3 Survival and proliferation of progenitors in brain-derived neurotrophic factor knock-in IV (BDNF-KIV) mice and effects of enriched environment (EE). Top panels: BrdU labeling paradigm. Middle panels: representative images displaying BrdU positive $(+)$ cells (arrows) from the four groups. Lower panels: quantification of BrdU + cells in the dentate gyrus (DG). (a) Number of surviving progenitor cells (3 weeks). Note the decreased number of surviving BrdU + cells in KIV-standard condition (SC) compared with wild-type (WT)-SC and the significant increase in the number of BrdU + cells in KIV-EE mice compared with both KIV-SC and WT-SC (18-20 DG in 9-10 sections from each brain, $n=5$ per group). (b) Number of proliferating cells ( $3 \mathrm{~h}$ ). Note the significant increase in the number of BrdU + cells in KIV-EE compared with KIV-SC and WT-SC (8-10 DG in 4-5 sections from each brain, $n=3$ per group). Results are expressed as $\%$ of WT-SC \pm s.e. Scale bar in (a) and $(\mathbf{b})=250 \mu \mathrm{m}$. ${ }^{*} P<0.05,{ }^{* \star} P<0.01$.

EE restored decreased BDNF protein levels in the HIP of BDNF-KIV mice.

Reduced survival of progenitor cells in BDNF-KIV mice that was reversed by EE. Next, we sought to determine the effect of EE on neurogenesis as a possible cellular mechanism underlying the depression-like behavior and its recovery in BDNF-KIV mice. Distinct stages of neurogenesis including the survival, proliferation and differentiation of newly born cells were examined using BrdU, a marker of dividing cells. When survival of BrdU + cells was examined following 3 weeks of housing in SC or EE (Figure 3, top panel), a significant effect of $\mathrm{EE}(P<0.01)$ and a genotypeEE interaction $(P<0.01)$ were detected. BDNF-KIV mice showed a significant decrease $(23 \%)$ in the number of $\mathrm{BrdU}+$ cells compared with WT mice in SC $(P<0.01$, Figure 3a) but not in $\mathrm{EE}(P=0.12)$. EE treatment significantly increased the number of BrdU + cells in BDNF-KIV mice 
$(P<0.01$, KIV-EE Vs KIV-SC) and WT mice $(P<0.05$, WT-EE vs WT-SC, Figure 3a). Strikingly, the increased rate of surviving progenitors by EE was greater in BDNF-KIV mice compared with WT mice (2.1-fold increase in BDNF-KIV mice vs 1.4-fold increase in WT mice, Student's $t$-test, $P<0.05)$. KIV-EE mice also showed a significant increase (1.6-fold) in the number of BrdU + cells compared with WT-SC $(P<0.01$, Figure 3a). These results indicate that EE not only reversed the reduction in the number of $\mathrm{BrdU}+$ cells observed in BDNF-KIV mice but also increased BrdU + cells more than the levels in WT mice.

\section{Normal proliferation in BDNF-KIV mice that was} increased by EE. Next, we examined whether the reduced $\mathrm{BrdU}+$ cell population in BDNF-KIV mice was the result of reduced progenitor proliferation. The number of $\mathrm{BrdU}+$ cells $3 \mathrm{~h}$ after BrdU injection was counted (Figure $3 \mathrm{~b}$, top panel); $3 \mathrm{~h}$ are sufficient for actively dividing cells to incorporate BrdU but not long enough to undergo the second round of mitosis. ${ }^{41}$ No difference was observed in the number of BrdU + cells between KIV-SC and WT-SC mice $(P=0.90$, Student's $t$-test, Figure $3 \mathrm{~b})$, indicating that the decreased number of BrdU + cells at 3 weeks observed in BDNF-KIV mice in SC (Figure 3a) was not the result of reduced proliferation but rather the result of reduced survival of the progenitors. Further, a significant effect of EE $(P<0.05)$ was detected. Remarkably, EE treatment significantly increased the number of $\mathrm{BrdU}+$ cells in BDNF-KIV mice compared with both BDNF-KIV mice in SC (1.4-fold, $P<0.05)$ and WT mice in SC $(1.4$-fold; $P<0.05$, Figure $3 b$ ), whereas EE had no effect on proliferation of progenitors in WT mice (WT-EE vs WT-SC; $P=0.28$, Student's $t$-test, Figure $3 b$ ). These data indicate that EE has an effect on DG cell proliferation in BDNF-KIV mice but not in WT mice and that the increased BrdU + cells after 3 weeks of EE in BDNF-KIV mice were the result of cumulative effects of $E E$ on both proliferation and survival of the progenitors.

Normal differentiation of progenitor cells in BDNF-KIV mice and EE had no influence on cell differentiation. Newly born cells in the DG either die or differentiate into neurons or glia. ${ }^{42}$ To determine the fate of newborn cells, we examined the percentage of $\mathrm{BrdU}+$ cells that acquired neuronal or glial phenotypes 3 weeks after the first BrdU injection, a time point at which most progenitors undergo terminal differentiation. ${ }^{42,43}$ Doublelabeling for BrdU and the neuronal marker NeuN or glial marker GFAP was performed. Most of the BrdU-stained cells (>90\%) were found to co-express NeuN, indicating a neuronal phenotype and no difference was observed in the differentiation of progenitors into NeuN + or GFAP + cells among the groups (BrdU $+/ \mathrm{NeuN}+$ : WT-SC, $92.9 \pm 0.7 \%$; KIV-SC, $92.0 \pm 0.7 \%$; WT-EE, $91.8 \pm 1.0 \%$; KIV-EE, $91.9 \pm 1.8 \%, P>0.05$ and BrdU +/GFAP + : WTSC, $3.5 \pm 0.6 \%$; KIV-SC, $5.5 \pm 0.9 \%$; WT-EE, $5.6 \pm 1.0$; KIVEE, $3.4 \pm 0.7, P>0.05$, Supplementary Table 2) These results indicate that lack of promoter IV-driven BDNF expression impaired the survival of the progenitors but did not affect the proportion of those cells differentiating into neurons or glia. The cell fate of DG progenitors was not affected by housing conditions (SC vs EE) in either genotype.

Dendritic arborization of newly born neurons was impaired in BDNF-KIV mice. Newly born neurons send dendrites to the molecular layer and receive synaptic input as part of the maturation process. ${ }^{42}$ We next examined the impact of promoter IV-driven BDNF expression on dendrite development of newly born neurons in the DG by using DCX, a marker of immature neurons. ${ }^{44} \mathrm{DCX}+$ cells in the DG exhibited dendrites that projected to the granule cell layer or crossed the granule cell layer to the molecular layer, with the axis of the body perpendicular to the SGZ in all groups (Figures $4 a$ and $b$ ). Quantification of the number of DCX + cells revealed no difference between WT and BDNF-KIV mice in SC ( $P=0.28$, Student's $t$-test, Figure 4c), indicating that cell survival was unchanged at this stage of neuronal development in BDNF-KIV mice. The extent of dendritic extension was then analyzed by counting the number of dendrites of DCX + cells that extended 50,100, 150 and $200 \mu \mathrm{m}$ from the SGZ. Dendritic extension was most commonly found up to $150 \mu \mathrm{m}$ from the SGZ in both genotypes. BDNF-KIV mice in SC exhibited a significantly reduced number of dendrites crossing at $50 \mu \mathrm{m}$ compared with WT-SC mice $\left(P_{(50 \mu m)}<0.01\right.$, Figure $\left.4 \mathrm{~d}\right)$.

EE reversed impaired dendritic extension of newly born neurons in BDNF-KIV mice. When the effect of EE on the number of DCX + cells was examined, two-way ANOVA revealed a significant effect of EE $(P<0.01)$ and genotype$\mathrm{EE}$ interaction $(P<0.01)$. EE significantly increased the number of DCX + cells in BDNF-KIV mice compared with BDNF-KIV mice in SC (1.9-fold, $P<0.01$, Figure $4 \mathrm{c})$, as well as compared with WT mice both in SC $(P<0.01)$ and EE $(P<0.01$, Figure $4 c)$. These data are in line with the results that EE increased cell proliferation and survival in BDNF-KIV mice. When the effect of $E E$ on dendritic extension was analyzed at 50, 100 and $150 \mu \mathrm{m}$ from the SGZ, effects of EE $\left(P_{(50 \mu \mathrm{m})}<0.05, P_{(100 \mu \mathrm{m})}<0.01, P_{(150 \mu \mathrm{m})}<0.01\right)$ and genotype $\left(P_{(50 \mu \mathrm{m})}<0.01, P_{(100 \mu \mathrm{m})}<0.05\right)$ were observed. EE significantly increased the number of dendrites in BDNF-KIV mice compared with those in SC at 50 and $100 \mu \mathrm{m}$ from the SGZ $\left(P_{(50 \mu \mathrm{m})}<0.01, \quad P_{(100 \mu \mathrm{m})}<0.01\right)$ and in both genotypes at $150 \mu \mathrm{m}$ (WT, $P<0.01 ; \quad \mathrm{KIV}, \quad P<0.01$; Figure 4d). Dendrites extending up to $200 \mu \mathrm{m}$ were observed in WT mice with EE treatment but not in other groups (Figures $4 a, b$ and d). No difference in the number of dendrites was observed between WT-EE and KIV-EE at any of these points (Figure $4 d$, Supplementary Table 2). These results indicate that EE reversed the reduced dendrite extension in BDNF-KIV mice and that EE increased the dendrite extension in both genotypes at the measurement points most distal from the SGZ.

No change in effect of EE on TUNEL + cells in the DG. To determine if the decreased or increased survival of progenitors in BDNF-KIV mice in SC or EE, respectively, was the result of increased or decreased apoptosis, we examined the number of TUNEL + apoptotic cells in the DG. The number of TUNEL + cells detected in the DG was low and 
a
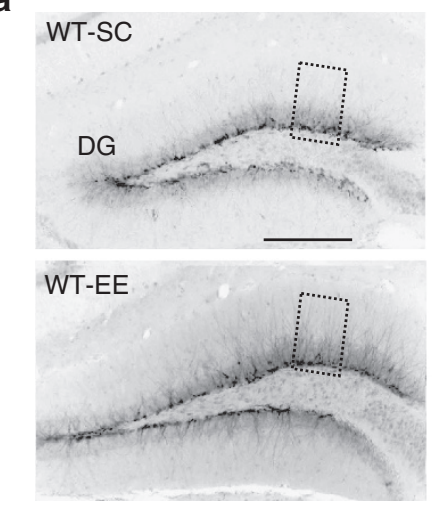

C

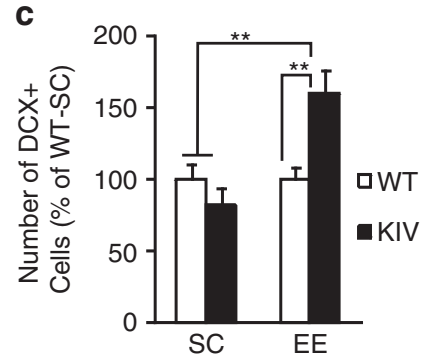

KIV-SC
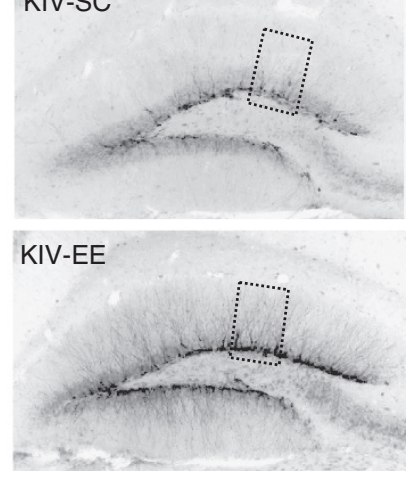

d

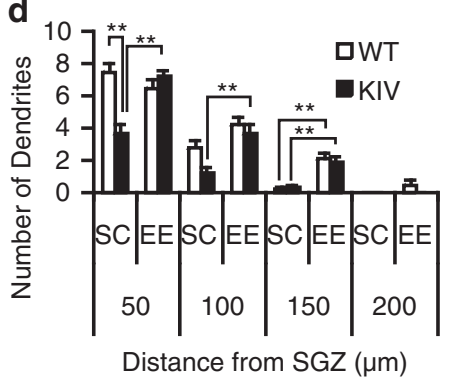

b

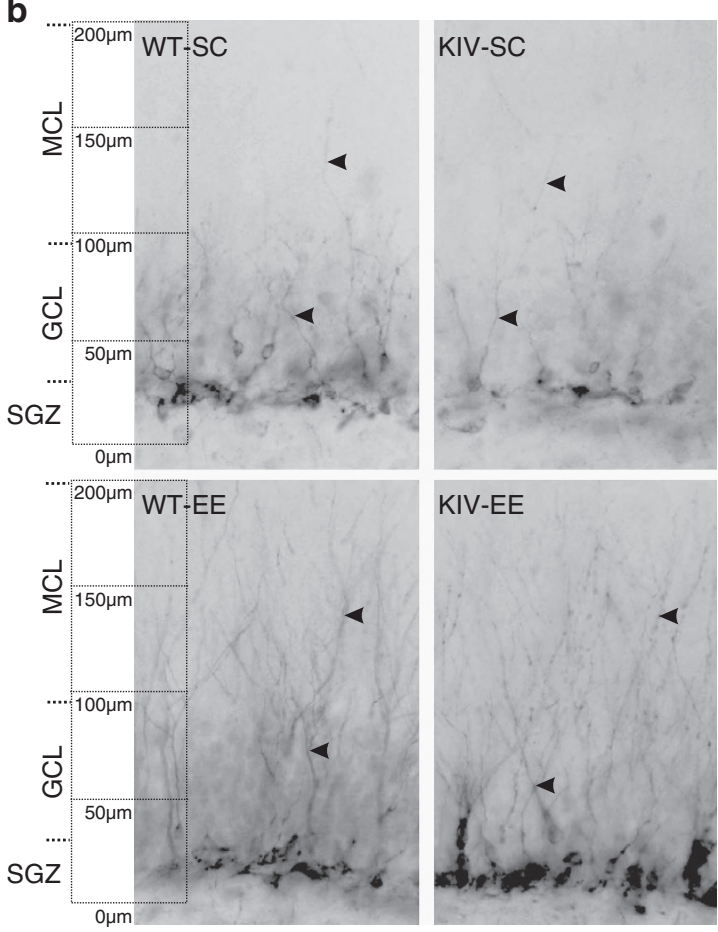

Figure 4 Doublecortin-positive (DCX +) cells and dendritic extension in the dentate gyrus (DG) and effect of enriched environment (EE). (a) Representative images showing DCX + cells and their dendrites from the four groups. Scale bar $=250 \mu \mathrm{m}$. (b) Magnified view of boxed area in (a) showing DCX + cells and their dendrites (arrowheads). (c) Quantification of DCX + cells in the DG. Note the significantly increased number of DCX + cells in knock-in IV (KIV)-EE mice compared with KIV-standard condition (SC) and wild-type (WT)-SC groups. Results are expressed as \% of WT-SC \pm s.e. (d) Quantification of the extension of apical dendrites of DCX + cells into the molecular cell layer of the DG. Note the significantly reduced numbers of dendrites in KIV-SC compared with WT-SC at $50 \mu \mathrm{m}$ from the subgranular zone (SGZ), which was rescued by EE. EE increased the number of dendrites of both genotypes at $150 \mu \mathrm{m}$ from the SGZ. (Two DG from each brain, $n=4$ per group). Results are expressed as mean \pm s.e. ${ }^{* *} P<0.01$.

was similar in all groups (WT-SC, $2.6 \pm 0.4$; KIV-SC, $2.8 \pm 0.5$; WT-EE, $3.2 \pm 0.5 ; \quad$ KIV-EE, $3.3 \pm 0.4$; values are mean \pm s.e.; $P>0.05$ for each comparison, one-way ANOVA).

\section{Discussion}

Results of this study demonstrated that 3 weeks of exposure to EE normalized decreased BDNF levels, decreased neurogenesis and depression-like behavior in a single line of mutant mice that lack promoter IV-driven BDNF expression. To our knowledge, this is the first study that shows these three factors (BDNF, neurogenesis and depression) correlated in a single animal model with a Bdnf gene manipulation. Our results showed that the depression-like phenotype caused by impaired promoter IV-driven BDNF expression could be reversed by EE treatment. EE treatment increased BDNF mRNA levels driven by BDNF promoters other than promoter IV and restored BDNF protein levels in the HIP of BDNF-KIV mice. This indicates that one promoter deficiency can be compensated by other multiple promoters in the Bdnf gene. Our results showed that lack of activitydependent promoter IV-driven BDNF leads to reduced survival and dendritic maturation of newly born neurons in the HIP. This suggests that reduced neurogenesis is one of the underlying mechanisms of depression-like behavior. EE treatment reversed decreased neurogenesis and BDNF levels, and restored depression-like behavior in BDNF-KIV mice. This suggests that increased BDNF and adult hippocampal neurogenesis may mediate the effects of $\mathrm{EE}$ on depression-like behavior. Whether increased BDNF levels and neurogenesis are sufficient to produce the entire effects of EE or whether they are parallel phenomena remain to be addressed.

In this study, we observed that EE normalized depressionlike behavior in BDNF-KIV mice, by increasing locomotor activity in the OFT, decreasing the immobility time in the TST and increasing sucrose consumption in the SPT, which is in line with the suggested antidepressant-like effect of EE. ${ }^{22,45}$ While the results in the OFT and TST suggest that EE increased overall locomotor activity, the results of the SPT indicate that EE rescue an anhedonia-like phenotype. EE did not change locomotor activity in the OFT in WT mice, which is similar to previous studies that have shown no effect of EE on locomotor activity in the OFT in WT rodents, ${ }^{46,47}$ although other studies have reported that EE reduced locomotor activity in the OFT. ${ }^{21,23,48}$ In the TST, we observed that EE also decreased immobility time of WT mice as well as of BDNF-KIV mice, which was similar to the previous studies that have shown exercise and antidepressant medications 
decreased immobility in WT mice..$^{23,39}$ In the SPT, EE did not increase sucrose preference of WT mice, which is similar to the previous reports where EE did not influence sucrose intake in control but showed recovery of reduced sucrose intake following chronic stress. ${ }^{45,49}$ In contrast, some of the effects (increased time in the center in the OFT and increased total liquid intake) of EE were observed only in WT mice. These results suggest that the behavioral effects of EE may be different depending on the behavioral baseline of the animal. To our knowledge, this is the first study to demonstrate that EE reverses the depression-like phenotype in a rodent model with reduced BDNF levels. Most other rodent models with reduced BDNF levels (for example, conditional BDNF KO mice, BDNF heterozygous mice and knockdown by injection with BDNF blockers) do not show depression-like behavior (for a review, see Duman and Monteggia ${ }^{3}$ ). A few studies have examined the antidepressant effects of EE on other rodent models with reduced BDNF levels; BDNF heterozygous mice have been reported to show no change in locomotor activity in the OFT after EE treatment ${ }^{47,48}$ and no change in immobility time in the forced swim test after voluntary exercise on a running wheel (an integral component of most EE paradigms), ${ }^{23}$ compared with those held in SC. The effects of EE may appear significant only to those mice that show depression-like behavior, and/or those retaining both alleles of the intact BDNF protein-coding region. A limited number of recent studies have shown depression-like behavior in rodents with reduced BDNF levels; ${ }^{20,50-52}$ however, the antidepressant-like effects of $E E$ on these rodent models have not yet been reported. The biggest difference between BDNF-KIV mice and other rodent models with reduced BDNF levels is that, in BDNF-KIV mice, the gene manipulation was made on an endogenous promoter without affecting other promoters and the protein-coding region of the Bdnf gene, while other rodent models lack some or all of the BDNF protein-coding region. In conditional $\mathrm{KO}$ mice, this deletion is regulated by promoters of other genes. Focusing on endogenous BDNF promoters may be critical to understanding the effects of the Bdnf gene regulation and antidepressant interventions, because epigenetic regulatory processes occur under stress and antidepressant treatments including EE. ${ }^{10,11,16,53}$

We observed that EE treatment increased total BDNF mRNA (exon IX) levels in the HIP of both BDNF-KIV and WT mice compared with those in SC. This increase was attributed to the increased levels of almost all exons, with significant effects of EE on exons I, II and III (1.5-2-fold). These results are partially consistent with another study that has shown exercise (4 weeks of running) induces relatively strong BDNF expression through promoters I, II and III (about two-fold) but not through promoters IV and VI in the HIP, while novel objects (for example, toys) without a running wheel induces moderate BDNF expression through promoter I, II, III, IV and VI (1.2-1.5-fold). ${ }^{16,54}$ Our results showing less effects of EE on promoter IV- and VI-driven BDNF transcripts suggests that exercise on a running wheel may be the leading cause of the induction of BDNF expression in our EE condition. One may expect that $E E$ will increase neuronal activity and will therefore induce activity-dependent promoter IV-driven BDNF expression. Therefore, no significant induction in promoter IV-driven
BDNF transcripts by EE may sound contradicting. However, this could be explained by the different time course of BDNF upregulation by different stimuli. For example, neuronal activity with calcium influx induced by glutamate and depolarization robustly induces promoter IV- and I-driven BDNF expression in an acute phase (3-6 h, usually $>6$-fold), while BDNF induction by EE, antidepressants, electroconvulsive therapy and exercise is not robust and requires longer time (1.2-3-fold increase over several weeks) and may involve cAMP (cyclic adenosine monophosphate) cascades (details in Sakata ${ }^{55}$ ), although the exact signaling pathways by which EE activates BDNF promoters remain to be addressed. It is interesting to note that EE induced BDNF mRNA through multiple promoters rather than through one or two promoters, which is in contrast to the effects of antidepressant medications that have been reported to use a relatively restricted set of BDNF promoters. For example, chronic desipramine treatment induces promoter I-driven BDNF expression, while chronic fluoxetine administration induces promoter II-driven BDNF expression in the HIP. ${ }^{14}$ EE may have stronger antidepressant effects than medications do by increasing expression of BDNF driven by multiple promoters, which can compensate the decreased function of promoter IV under stress and lack of neuronal activity.

The increased BDNF mRNA levels by EE treatment were reflected in BDNF protein levels. Although BDNF-KIV mice in SC showed significantly reduced BDNF protein levels compared with WT mice in SC, this reduction was restored to levels similar to those in WT mice in SC. Reports have shown that 30 days or 8 weeks of EE did not increase hippocampal BDNF protein levels in BDNF heterozygous mice $^{56}$ or CaMKII promoter-conditional BDNF KO mice. ${ }^{57}$ Although the exact reason for this difference in BDNF protein induction by $E E$ is not clear, it can be speculated that both alleles of the Bdnf gene including the protein region may be necessary to induce BDNF protein levels. We observed that EE treatment did not significantly increase BDNF protein levels in WT mice. Reports have shown that 8 weeks, ${ }^{56}$ but not 30 days, ${ }^{57}$ of EE treatment resulted in increased hippocampal BDNF protein levels in WT rodents. It is possible that longer EE treatment may be required to significantly increase BDNF protein levels in WT mice.

Our results showed that BDNF-KIV mice showed a decrease in surviving progenitors but no change in cell proliferation or differentiation as compared with WT. This indicates that promoter IV-driven BDNF expression is critical for long-term survival of progenitors but not for proliferation or differentiation. Studies using BDNF mutant mice have reported conflicting results regarding the effects of reduced BDNF levels on neurogenesis: BDNF heterozygotes and conditional $\mathrm{KO}$ mice with a $\sim 50 \%$ reduction in BDNF levels compared with WT have shown either decreased ${ }^{57,58}$ or unchanged $^{56}$ survival of progenitors, with decreased, ${ }^{59}$ increased $^{58}$ or unchanged ${ }^{57}$ proliferation of progenitors. A recent study showed BDNF knockdown in the dorsal DG causes a significant reduction in differentiation of newborn progenitors into immature neurons without affecting proliferation and cell survival measured at $24 \mathrm{~h}$ and 7 days after BrdU injections. ${ }^{20}$ It is not clear whether these differences are attributed to methodological differences or to different BDNF 
levels in the HIP. Our results showing decreased dendritic length of DCX immunoreactivity in BDNF-KIV mice indicate that promoter IV-driven BDNF is critical for dendritic development during adult neurogenesis. This idea is supported by other studies that have shown reduced arborization in conditional BDNF KO mice under the CaMKII-promoter. ${ }^{57,60}$ Overall, our results showed a correlation between reduced neurogenesis in the HIP and depression-like behavior in promoter IV-deficient mice.

Three weeks of EE treatment increased survival of progenitors in both BDNF-KIV and WT mice without changing the neuron-glia ratio. Strikingly, the effect of EE on the survival of progenitors was significantly greater in BDNF-KIV mice (2.1-fold increase) than in WT mice (1.4-fold increase). This may be attributed to increased proliferation of progenitors stimulated by EE in BDNF-KIV mice, as well as increased survival rates, while proliferation was unaffected in WT mice. It is not clear why the effects of EE on neurogenesis are larger in BDNF-KIV mice compared with WT mice. Promoter IV deficiency did not enhance compensational expression of BDNF through other promoters as BDNF-KIV mice showed no difference in RNA induction rate and BDNF protein levels compared with WT-SC and WT-EE (Figure 2). One possibility is that the effect of EE may be enhanced by compensational mechanisms, such as increased availability of the BDNF receptor, TrkB, in BDNF-KIV mice where basal levels of BDNF are low. Interestingly, a study has implied a similar homeostatic regulation of BDNF in neurogenesis: long-term delivery of exogenous BDNF in the DG attenuated an ischemia-induced increase in neurogenesis, suggesting that increased BDNF levels reduce the intrinsic neuroregenerative response. ${ }^{61}$ Conversely, the reduced basal BDNF levels in BDNF-KIV mice may increase the neuroregenerative response. Effects of increased BDNF levels might be different between normal and abnormal brains. Our study also showed that EE treatment increased dendritic length of DG neurons in both WT and BDNF-KIV mice, indicating its effect of enhancing neuronal maturation and integration. Taken together, our results suggest that EE treatment increases the population of functional newly born neurons in the DG.

The antidepressant-like effects of EE tested in several behavioral paradigms, including the TST, are coupled with a significant increase in neurogenesis in the HIP. ${ }^{21,22,49}$ These consistent observations suggest a correlation between the antidepressant-like effects of EE and increased neurogenesis. However, it is still unclear whether increased adult neurogenesis is mediating the EE-induced antidepressantlike effects or whether the EE-induced antidepressant-like behavioral change leads to increased neurogenesis. A study has suggested that hippocampal adult neurogenesis is not required for the antidepressant and anti-anxiety effects of EE, because blocking neurogenesis with hippocampal X-irradiation did not alter EE-induced reduction of latency to food in the novelty suppressed feeding test. ${ }^{62}$ As BDNF enhances synaptic plasticity, ${ }^{63}$ it is possible that EE treatment causes its antidepressant effects without neurogenesis by increasing BDNF levels and enhancing the synaptic plasticity of existing mature cells. On the other hand, a recent study suggested that neurogenesis is required for the behavioral effects of EE after psychosocial stress exposure because the antidepressant effects of EE in the SPT were not observed when adult neurogenesis was selectively ablated by ganciclovir (a toxin to proliferating cells). ${ }^{64}$ The difference in these results may be attributed to methodological differences; however, these studies suggest that adult neurogenesis is required for EE-induced antidepressant-like effects only when under a stress condition but not in the basal condition. BDNF-KIV mice lacking promoter IV-driven BDNF expression may mimic the stress condition because promoter IV activity is decreased by different types of stress. ${ }^{10,11}$

Whether endogenous induction of BDNF levels in the HIP by $E E$ is sufficient and necessary to increase neurogenesis and produce antidepressant effects is yet to be determined. However, studies have suggested this causal relationship by showing that direct infusion of the BDNF protein into the HIP produced increased neurogenesis ${ }^{2}$ and antidepressant-like responses in several behavioral paradigms. ${ }^{18}$ Interestingly, a more recent study demonstrated that even peripheral BDNF administration $^{65}$ increased neurogenesis in the HIP and produced an antidepressant-like effect. In addition, a study has reported that deleting the BDNF receptor, TrkB, specifically in hippocampal neuronal progenitor cells was sufficient to blunt the effects of antidepressant medications. ${ }^{66}$ This suggests that BDNF-TrkB signaling is required for the antidepressant-like behavioral effects of these interventions. It will be interesting to see whether increased BDNF levels through other promoters inducted by $E E$ is required for the antidepressant effect in BDNF-KIV mice in the future by testing whether the EE-induced antidepressant effect can be blocked by specific BDNF-TrkB signaling inhibitors (for example, TrkB-blocking antibody) or RNA interference methods. Alternatively, the increase in neurogenesis and antidepressant effects of EE in BDNF-KIV mice may occur independently regardless of increased BDNF levels by recruiting other growth factors and monoamines. For example, vascular endothelial growth factor, ${ }^{67}$ insulin-like growth factor- $1,{ }^{68}$ serotonin and norepinephrine ${ }^{69}$ are upregulated by EE and have been shown to provide antidepressant-like effects, ${ }^{70,71}$ where vascular endothelial growth factor ${ }^{67}$ and insulin-like growth factor ${ }^{68}$ also increase neurogenesis. Whether these factors are involved in the EE-induced antidepressant effects in BDNF-KIV mice remains to be examined. Either way, from a therapeutic point of view, it is exciting to note that the depression-like behavior caused by the lack of promoter IV-driven BDNF expression can be reversed by EE treatment.

In conclusion, 3 weeks of EE treatment normalized the depression-like behavior, reduced BDNF protein levels and decreased neurogenesis in the HIP exhibited by BDNF-KIV mice. These results provide critical evidence for a pathophysiology of depression-like behavior-impaired promoter IV-driven BDNF expression results in decreased neurogenesis-and a possible mechanism of recovery; increased neurogenesis in the HIP by EE through increased BDNF levels driven by multiple BDNF promoters.

\section{Conflict of interest}

The authors declare no conflict of interest. 
Acknowledgements. We thank Drs Jeff D. Steketee and David L Armbruster for thoughtful comments and advice on, and editing of, the manuscript.

1. Thoenen H. Neurotrophins and neuronal plasticity. Science 1995; 270: 593-598.

2. Scharfman H, Goodman J, Macleod A, Phani S, Antonelli C, Croll S. Increased neurogenesis and the ectopic granule cells after intrahippocampal BDNF infusion in adult rats. Exp Neurol 2005; 192: 348-356.

3. Duman RS, Monteggia LM. A neurotrophic model for stress-related mood disorders. Biol Psychiatry 2006; 59: 1116-1127.

4. Sakata K, Woo NH, Martinowich K, Greene JS, Schloesser RJ, Shen L et al. Critical role of promoter IV-driven BDNF transcription in GABAergic transmission and synaptic plasticity in the prefrontal cortex. Proc Natl Acad Sci U S A 2009; 106: 5942-5947.

5. Sakata K, Jin L, Jha S. Lack of promoter IV-driven BDNF transcription results in depression-like behavior. Genes Brain Behav 2010; 26: 712-721.

6. Aid T, Kazantseva A, Piirsoo M, Palm K, Timmusk T. Mouse and rat BDNF gene structure and expression revisited. J Neurosci Res 2007; 85: 525-535.

7. Timmusk T, Palm K, Metsis M, Reintam T, Paalme V, Saarma M et al. Multiple promoters direct tissue-specific expression of the rat BDNF gene. Neuron 1993; 10: 475-489

8. Tao X, Finkbeiner S, Arnold DB, Shaywitz AJ, Greenberg ME. Ca2+ influx regulates BDNF transcription by a CREB family transcription factor-dependent mechanism. Neuron 1998; 20: 709-726

9. Shieh $\mathrm{PB}, \mathrm{Hu} \mathrm{SC}$, Bobb K, Timmusk T, Ghosh A. Identification of a signaling pathway involved in calcium regulation of BDNF expression. Neuron 1998; 20: 727-740.

10. Tsankova NM, Berton O, Renthal W, Kumar A, Neve RL, Nestler EJ. Sustained hippocampal chromatin regulation in a mouse model of depression and antidepressant action. Nat Neurosci 2006; 9: 519-525.

11. Fuchikami M, Morinobu S, Kurata A, Yamamoto S, Yamawaki S. Single immobilization stress differentially alters the expression profile of transcripts of the brain-derived neurotrophic factor (BDNF) gene and histone acetylation at its promoters in the rat hippocampus. Int J Neuropsychopharmacol 2009; 12: 73-82.

12. Adlard PA, Perreau VM, Engesser-Cesar C, Cotman CW. The timecourse of induction of brain-derived neurotrophic factor mRNA and protein in the rat hippocampus following voluntary exercise. Neurosci Lett 2004; 363: 43-48.

13. Dias BG, Banerjee SB, Duman RS, Vaidya VA. Differential regulation of brain derived neurotrophic factor transcripts by antidepressant treatments in the adult rat brain. Neuropharmacology 2003; 45: 553-563.

14. Dwivedi Y, Rizavi HS, Pandey GN. Antidepressants reverse corticosterone-mediated decrease in brain-derived neurotrophic factor expression: differential regulation of specific exons by antidepressants and corticosterone. Neuroscience 2006; 139: 1017-1029.

15. Khundakar AA, Zetterstrom TS. Biphasic change in BDNF gene expression following antidepressant drug treatment explained by differential transcript regulation. Brain Res 2006; 1106: 12-20.

16. Zajac MS, Pang TY, Wong N, Weinrich B, Leang LS, Craig JM et al. Wheel running and environmental enrichment differentially modify exon-specific BDNF expression in the hippocampus of wild-type and pre-motor symptomatic male and female Huntington's disease mice. Hippocampus 2010; 20: 621-636.

17. Metsis M, Timmusk T, Arenas E, Persson $H$. Differential usage of multiple brain-derived neurotrophic factor promoters in the rat brain following neuronal activation. Proc Natl Acad Sci U S A 1993; 90: 8802-8806.

18. Shirayama Y, Chen AC, Nakagawa S, Russell DS, Duman RS. Brain-derived neurotrophic factor produces antidepressant effects in behavioral models of depression. $J$ Neurosci 2002; 22: 3251-3261.

19. Groves JO. Is it time to reassess the BDNF hypothesis of depression? Mol Psychiatry 2007; 12: 1079-1088.

20. Taliaz D, Stall N, Dar DE, Zangen A. Knockdown of brain-derived neurotrophic factor in specific brain sites precipitates behaviors associated with depression and reduces neurogenesis. Mol Psychiatry 2010; 15: 80-92.

21. Hattori S, Hashimoto R, Miyakawa T, Yamanaka H, Maeno H, Wada $\mathrm{K}$ et al. Enriched environments influence depression-related behavior in adult mice and the survival of newborn cells in their hippocampi. Behav Brain Res 2007; 180: 69-76.

22. Xu Z, Hou B, Zhang Y, Gao Y, Wu Y, Zhao S et al. Antidepressive behaviors induced by enriched environment might be modulated by glucocorticoid levels. Eur Neuropsychopharmacol 2009; 19: 868-875.

23. Duman $\mathrm{CH}$, Schlesinger L, Russell DS, Duman RS. Voluntary exercise produces antidepressant and anxiolytic behavioral effects in mice. Brain Res 2008; 1199: 148-158.

24. Sahay A. Hen R. Adult hippocampal neurogenesis in depression. Nat Neurosci 2007; 10 $1110-1115$.

25. Gould E, Tanapat P. Stress and hippocampal neurogenesis. Biol Psychiatry 1999; 46: 1472-1479.

26. Malberg JE, Eisch AJ, Nestler EJ, Duman RS. Chronic antidepressant treatment increases neurogenesis in adult rat hippocampus. J Neurosci 2000; 20: 9104-9110.

27. van Praag $H$, Kempermann G, Gage FH. Running increases cell proliferation and neurogenesis in the adult mouse dentate gyrus. Nat Neurosci 1999; 2: 266-270.

28. Kempermann G, Kuhn HG, Gage FH. More hippocampal neurons in adult mice living in an enriched environment. Nature 1997; 386: 493-495.
29. Nibuya M, Takahashi M, Russell DS, Duman RS. Repeated stress increases catalytic TrkB mRNA in rat hippocampus. Neurosci Lett 1999; 267: 81-84.

30. Gronli J, Bramham C, Murison R, Kanhema T, Fiske E, Bjorvatn B et al. Chronic mild stress inhibits BDNF protein expression and CREB activation in the dentate gyrus but not in the hippocampus proper. Pharmacol Biochem Behav 2006; 85: 842-849.

31. Smith MA, Makino S, Kvetnansky R, Post RM. Stress and glucocorticoids affect the expression of brain-derived neurotrophic factor and neurotrophin-3 mRNAs in the hippocampus. J Neurosci 1995; 15: 1768-1777.

32. Ueyama T, Kawai Y, Nemoto K, Sekimoto M, Tone S, Senba E. Immobilization stress reduced the expression of neurotrophins and their receptors in the rat brain. Neurosci Res 1997; 28: 103-110.

33. Rasmusson AM, Shi L, Duman R. Downregulation of BDNF mRNA in the hippocampal dentate gyrus after re-exposure to cues previously associated with footshock. Neuropsychopharmacology 2002; 27: 133-142.

34. Pizarro JM, Lumley LA, Medina W, Robison CL, Chang WE, Alagappan A et al. Acute social defeat reduces neurotrophin expression in brain cortical and subcortical areas in mice. Brain Res 2004; 1025: 10-20.

35. Schmidt HD, Duman RS. The role of neurotrophic factors in adult hippocampa neurogenesis, antidepressant treatments and animal models of depressive-like behavior. Behav Pharmacol 2007; 18: 391-418.

36. Livak KJ, Schmittgen TD. Analysis of relative gene expression data using real-time quantitative PCR and the 2(-delta delta C(T)) method. Methods 2001; 25: 402-408

37. Shimazu K, Zhao M, Sakata K, Akbarian S, Bates B, Jaenisch R et al. NT-3 facilitates hippocampal plasticity and learning and memory by regulating neurogenesis. Learn Mem 2006; 13: 307-315

38. Walsh RN, Cummins RA. The open-field test: a critical review. Psychol Bull 1976; 83 482-504.

39. Cryan JF, Mombereau C, Vassout A. The tail suspension test as a model for assessing antidepressant activity: review of pharmacological and genetic studies in mice. Neurosci Biobehav Rev 2005; 29: 571-625.

40. Pothion S, Bizot JC, Trovcro F, Belzung C. Strain differences in sucrose preference and in the consequences of unpredictable chronic mild stress. Behav Brain Res 2004; 155: 135 146.

41. Cameron HA, McKay RD. Adult neurogenesis produces a large pool of new granule cells in the dentate gyrus. J Comp Neurol 2001; 435: 406-417.

42. Kempermann G, Jessberger S, Steiner B, Kronenberg G. Milestones of neuronal development in the adult hippocampus. Trends Neurosci 2004; 27: 447-452.

43. Brandt MD, Jessberger S, Steiner B, Kronenberg G, Reuter K, Bick-Sander A et al. Transient calretinin expression defines early postmitotic step of neuronal differentiation in adult hippocampal neurogenesis of mice. Mol Cell Neurosci 2003; 24: 603-613.

44. Brown JP, Couillard-Despres S, Cooper-Kuhn CM, Winkler J, Aigner L, Kuhn HG Transient expression of doublecortin during adult neurogenesis. J Comp Neurol 2003; 467 $1-10$.

45. Green TA, Alibhai IN, Roybal CN, Winstanley CA, Theobald DE, Birnbaum SG et al. Environmental enrichment produces a behavioral phenotype mediated by low cyclic adenosine monophosphate response element binding (CREB) activity in the nucleus accumbens. Biol Psychiatry 2010; 67: 28-35.

46. Chourbaji S, Zacher C, Sanchis-Segura C, Spanagel R, Gass P. Social and structural housing conditions influence the development of a depressive-like phenotype in the learned helplessness paradigm in male mice. Behav Brain Res 2005; 164 100-106.

47. Chourbaji S, Brandwein C, Vogt MA, Dormann C, Hellweg R, Gass P. Nature vs nurture: can enrichment rescue the behavioural phenotype of BDNF heterozygous mice? Behav Brain Res 2008; 192: 254-258.

48. Zhu SW, Codita A, Bogdanovic N, Hjerling-Leffler J, Ernfors P, Winblad B et al. Influence of environmental manipulation on exploratory behaviour in male BDNF knockout mice. Behav Brain Res 2009; 197: 339-346.

49. Veena J, Srikumar BN, Raju TR, Shankaranarayana Rao BS. Exposure to enriched environment restores the survival and differentiation of new born cells in the hippocampus and ameliorates depressive symptoms in chronically stressed rats. Neurosci Lett 2009; 455: 178-182.

50. Chan JP, Unger TJ, Byrnes J, Rios M. Examination of behavioral deficits triggered by argeting BDNF in fetal or postnatal brains of mice. Neuroscience 2006; 142: 49-58.

51. Monteggia LM, Luikart B, Barrot M, Theobold D, Malkovska I, Nef S et al. Brain-derived neurotrophic factor conditional knockouts show gender differences in depression-related behaviors. Biol Psychiatry 2007; 61: 187-197.

52. Autry AE, Adachi M, Cheng P, Monteggia LM. Gender-specific impact of brain-derived neurotrophic factor signaling on stress-induced depression-like behavior. Biol Psychiatry 2009; 66: 84-90

53. Kuzumaki N, Ikegami D, Tamura R, Hareyama N, Imai S, Narita M et al. Hippocampal epigenetic modification at the brain-derived neurotrophic factor gene induced by an enriched environment. Hippocampus 2011; 21: 127-132.

54. Russo-Neustadt AA, Beard RC, Huang YM, Cotman CW. Physical activity and antidepressant treatment potentiate the expression of specific brain-derived neurotrophic factor transcripts in the rat hippocampus. Neuroscience 2000; 101: 305-312.

55. Sakata K. Gene regulation of BDNF and major depression. In press: Francisco L-M (ed). Neurobiology of Depression. CRC press: FL, USA, 2011 
56. Rossi C, Angelucci A, Costantin L, Braschi C, Mazzantini M, Babbini F et al. Brain-derived neurotrophic factor (BDNF) is required for the enhancement of hippocampal neurogenesis following environmental enrichment. Eur J Neurosci 2006; 24: 1850-1856.

57. Choi SH, Li Y, Parada LF, Sisodia SS. Regulation of hippocampal progenitor cell survival, proliferation and dendritic development by BDNF. Mol Neurodegener 2009; 4: 52.

58. Sairanen M, Lucas G, Ernfors P, Castren M, Castren E. Brain-derived neurotrophic factor and antidepressant drugs have different but coordinated effects on neuronal turnover, proliferation, and survival in the adult dentate gyrus. $J$ Neurosci 2005; 25 . 1089-1094

59. Lee J, Duan W, Mattson MP. Evidence that brain-derived neurotrophic factor is required for basal neurogenesis and mediates, in part, the enhancement of neurogenesis by dietary restriction in the hippocampus of adult mice. J Neurochem 2002; 82: 1367-1375.

60. Chan JP, Cordeira J, Calderon GA, Iyer LK, Rios M. Depletion of central BDNF in mice impedes terminal differentiation of new granule neurons in the adult hippocampus. Mol Cell Neurosci 2008; 39: 372-383.

61. Larsson E, Mandel RJ, Klein RL, Muzyczka N, Lindvall O, Kokaia Z. Suppression of insultinduced neurogenesis in adult rat brain by brain-derived neurotrophic factor. Exp Neurol 2002; 177: 1-8.

62. Meshi D, Drew MR, Saxe M, Ansorge MS, David D, Santarelli L et al. Hippocampal neurogenesis is not required for behavioral effects of environmental enrichment. Nat Neurosci 2006; 9: 729-731.

63. Lu B, Chow A. Neurotrophins and hippocampal synaptic transmission and plasticity. J Neurosci Res 1999; 58: 76-87.

64. Schloesser RJ, Lehmann M, Martinowich K, Manji HK, Herkenham M. Environmenta enrichment requires adult neurogenesis to facilitate the recovery from psychosocial stress. Mol Psychiatry 2010; 15: 1152-1163.
65. Schmidt HD, Duman RS. Peripheral BDNF produces antidepressant-like effects in cellular and behavioral models. Neuropsychopharmacology 2010; 35: 2378-2391.

66. Li Y, Luikart BW, Birnbaum S, Chen J, Kwon CH, Kernie SG et al. TrkB regulates hippocampal neurogenesis and governs sensitivity to antidepressive treatment. Neuron 2008; 59: 399-412

67. Cao L, Jiao X, Zuzga DS, Liu Y, Fong DM, Young D et al. VEGF links hippocampal activity with neurogenesis, learning and memory. Nat Genet 2004; 36: 827-835.

68. Trejo JL, Carro E, Torres-Aleman I. Circulating insulin-like growth factor I mediates exercise-induced increases in the number of new neurons in the adult hippocampus. J Neurosci 2001; 21: 1628-1634.

69. Brenes JC, Padilla M, Fornaguera J. A detailed analysis of open-field habituation and behavioral and neurochemical antidepressant-like effects in postweaning enriched rats. Behav Brain Res 2009; 197: 125-137.

70. Duman $\mathrm{CH}$, Schlesinger L, Terwilliger R, Russell DS, Newton SS, Duman RS. Periphera insulin-like growth factor-I produces antidepressant-like behavior and contributes to the effect of exercise. Behav Brain Res 2009; 198: 366-371.

71. Warner-Schmidt JL, Duman RS. VEGF is an essential mediator of the neurogenic and behavioral actions of antidepressants. Proc Natl Acad Sci U S A 2007; 104: 4647-4652.

Translational Psychiatry is an open-access journal published by Nature Publishing Group. This work is licensed under the Creative Commons Attribution-Noncommercial-Share Alike 3.0 Unported License. To view a copy of this license, visit http:// creativecommons.org/licenses/by-nc-sa/3.0/

\section{Supplementary Information accompanies the paper on the Translational Psychiatry website (http://www.nature.com/tp)}

